\title{
Caudal Duplication Syndrome: the Vital Role of a Multidisciplinary Approach and Staged Correction
}

\author{
Inbal Samuk ${ }^{1}$ Marc Levitt ${ }^{2}$ Elena Dlugy ${ }^{1}$ Dragan Kravarusic ${ }^{1}$ David Ben-Meir ${ }^{3}$ Gustavo Rajz ${ }^{4}$ \\ Osnat Konen $^{5}$ Enrique Freud ${ }^{1}$
}

\footnotetext{
${ }^{1}$ Department of Pediatric Surgery, Schneider Children's Medical Center, Sackler Medical School, University of Tel Aviv, Petach Tikvah, Israel

${ }^{2}$ Center for Colorectal and Pelvic Reconstruction, Nationwide Children's Hospital, Columbus, Ohio, United States

3 Pediatric Urology Unit, Schneider Children's Medical Center, Sackler Medical School, University of Tel Aviv, Petach Tikvah, Israel

4 Pediatric Neurosurgery Unit, Schneider Children's Medical Center, Sackler Medical School, University of Tel Aviv, Petach Tikvah, Israel

5 Radiology Department, Schneider Children's Medical Center, Sackler Medical School, University of Tel Aviv, Petach Tikvah, Israel
}

\begin{abstract}
Address for correspondence Inbal Samuk, MD, Department of Pediatric Surgery, Schneider Children's Medical Center, 14 Kaplan Street, Petach Tikvah 49202, Israel (e-mail: inbalsabl@clalit.org.il).
\end{abstract}

Eur J Pediatr Surg Rep 2016;4:1-5.

\author{
Abstract \\ Keywords \\ - caudal duplication \\ syndrome \\ - anorectal \\ malformation \\ - rectal duplication \\ - colonic duplication \\ - genitourinary \\ duplication
}

Caudal duplication syndrome is a rare entity that describes the association between congenital anomalies involving caudal structures and may have a wide spectrum of clinical manifestations. A full-term male presented with combination of anomalies including anorectal malformation, duplication of the colon and lower urinary tract, split of the lower spine, and lipomyelomeningocele with tethering of the cord. We report this exceptional case of caudal duplication syndrome with special emphasis on surgical strategy and approach combining all disciplines involved. The purpose of this report is to present the pathology, assessment, and management strategy of this complex case.

\section{New Insights and the Importance for the Pediatric Surgeon}

Caudal duplication syndrome (CDS) is a rare, complex entity that mandates careful assessment. The surgical correction requires a clear preestablished staged approach combining all disciplines involved.

\section{Introduction}

CDS represents a rare entity of congenital anomalies that involve the gastrointestinal and urogenital tracts and neural tube. ${ }^{1,2}$ The term caudal duplication described a spectrum in which structures derived from the embryonic cloaca and notochord are duplicated to various degrees. ${ }^{3}$ Within that spectrum, the clinical presentation of each patient varies considerably. Therefore, the surgical approach should be planned and tailored individually using a multidisciplinary team approach, taking into consideration the diverse aspects of the anomaly, to optimally prioritize their management.

We report an exceptional case of a male baby with CDS and delineate the surgical approach and objectives.
License terms

Stuttgart · New York

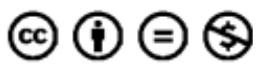

August 16, 2015

accepted after revision

November 9, 2015

published online

December 31, 2015

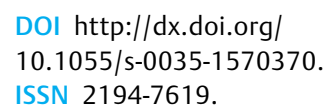




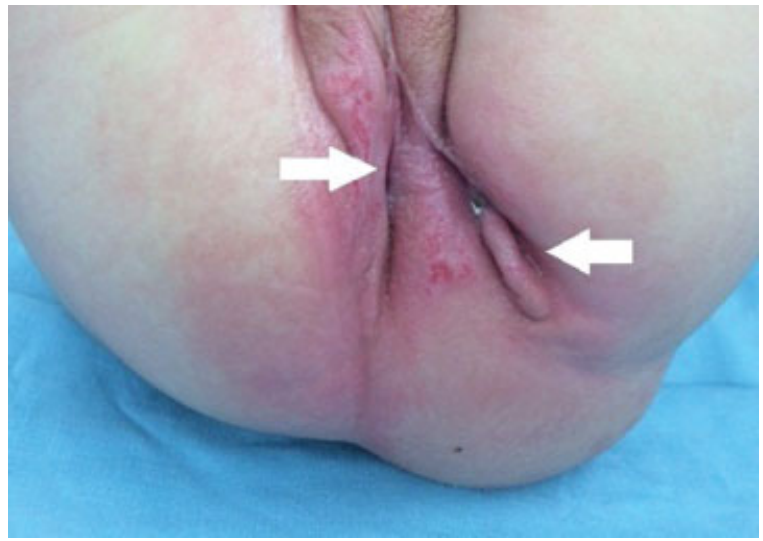

Fig. 1 Perineum showing two perineal fistulas (arrows). The right perineal fistula is located in the midline groove, which is pushed to the right by the sacral lump.

\section{Case Report}

A 2,665-g full-term male, born by vaginal delivery following an uneventful pregnancy, was evaluated for associated anomalies. There was no background of parental consanguinity, and a prenatal assessment was not performed due to religious beliefs. Physical examination after birth revealed two perineal fistulae, two external urethral orifices that opened separately along the penile shaft, a bifid scrotum, lower spinal deformity, a soft lump in the sacral area with hemangioma, and normal lower limbs. One of the perineal fistulae was located in the midline, which was pushed to the right by the sacral lump, and the other fistula was located left lateral to it (-Fig. 1). The baby was stooling from both perineal orifices and passed urine from both urethras during micturition. Plain lumbosacral radiograph showed severe dysplasia of lumbo-

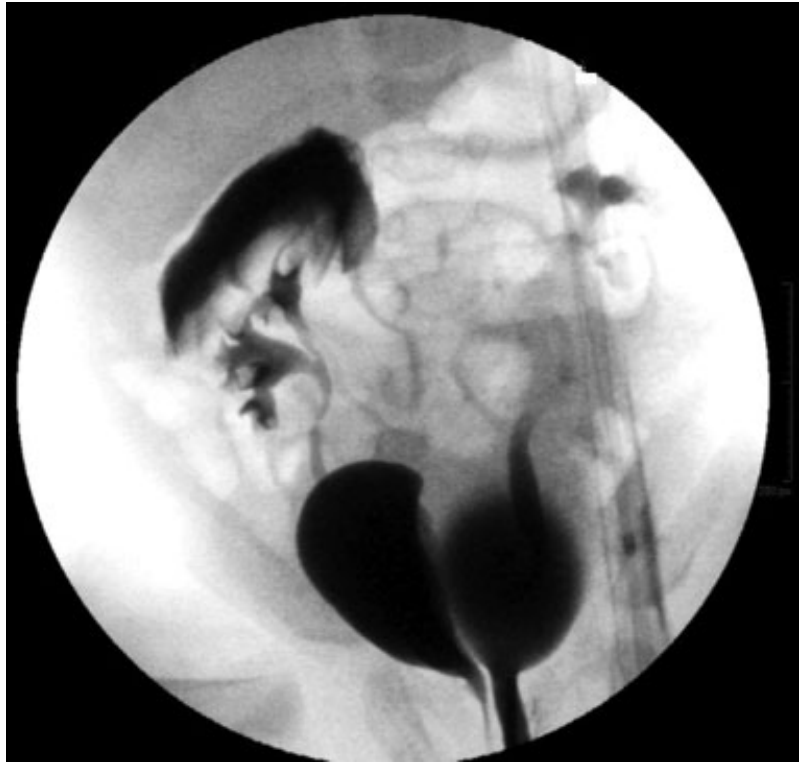

Fig. 3 Retrograde cystourethrogram showing two hemibladders and two completely separated urethras.

sacral vertebral spine. Contrast study through both perineal fistulae demonstrated duplication of the rectum and colon to the level of the hepatic flexure, although it was difficult to define the exact extension level of duplication ( - Fig. 2). Renal ultrasound showed normal kidneys with mild left hydronephrosis. The urinary tract was further evaluated by cystoscopy, retrograde cystourethrogram, and a cystometric study. On retrograde cystourethrogram, two hemibladders were clearly noted with grade II left vesicoureteral reflux (-Fig. 3). On cystoscopy, both urethras were normal. The distal penile urethra led to a small left hemibladder with
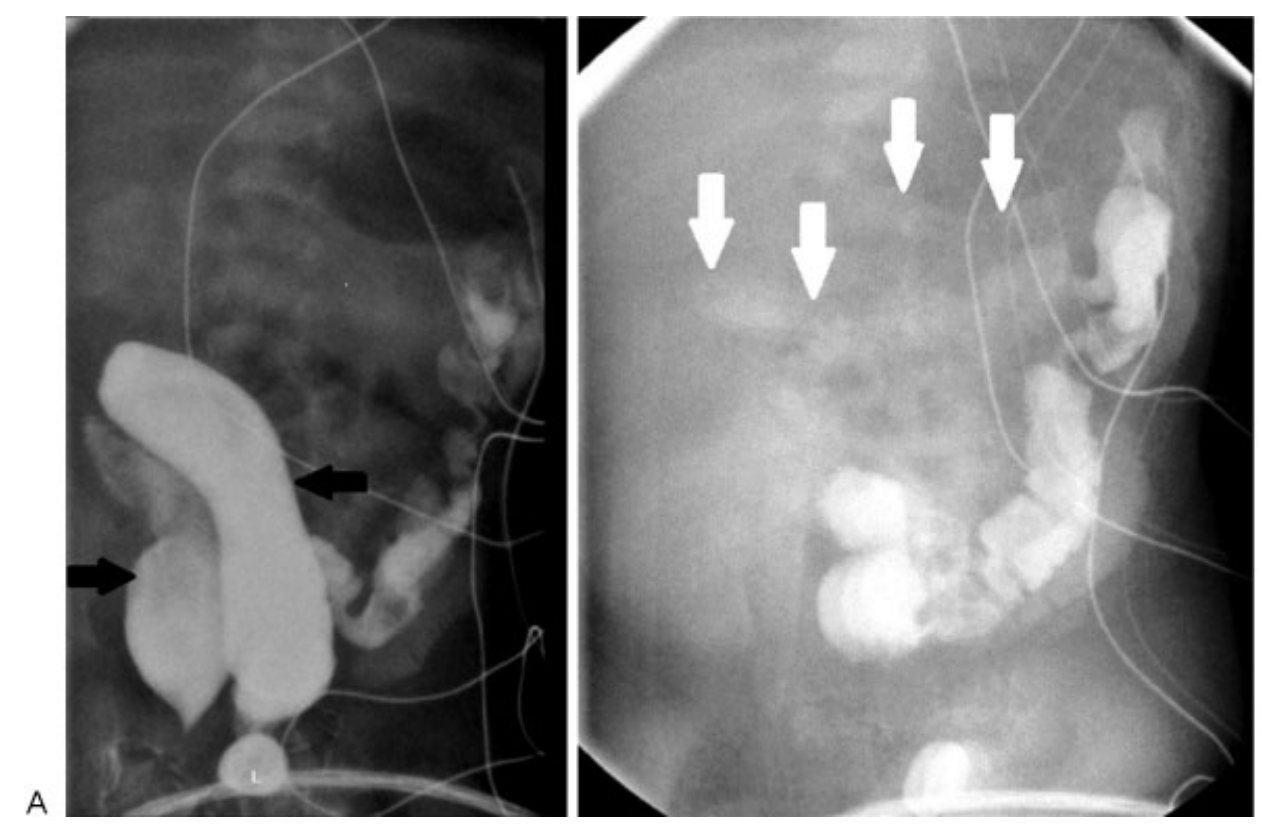

B

Fig. 2 Contrast study through both perineal fistulae demonstrates duplication of the (A) rectum (black arrows) and (B) colon (white arrows) to the level of hepatic flexure. 


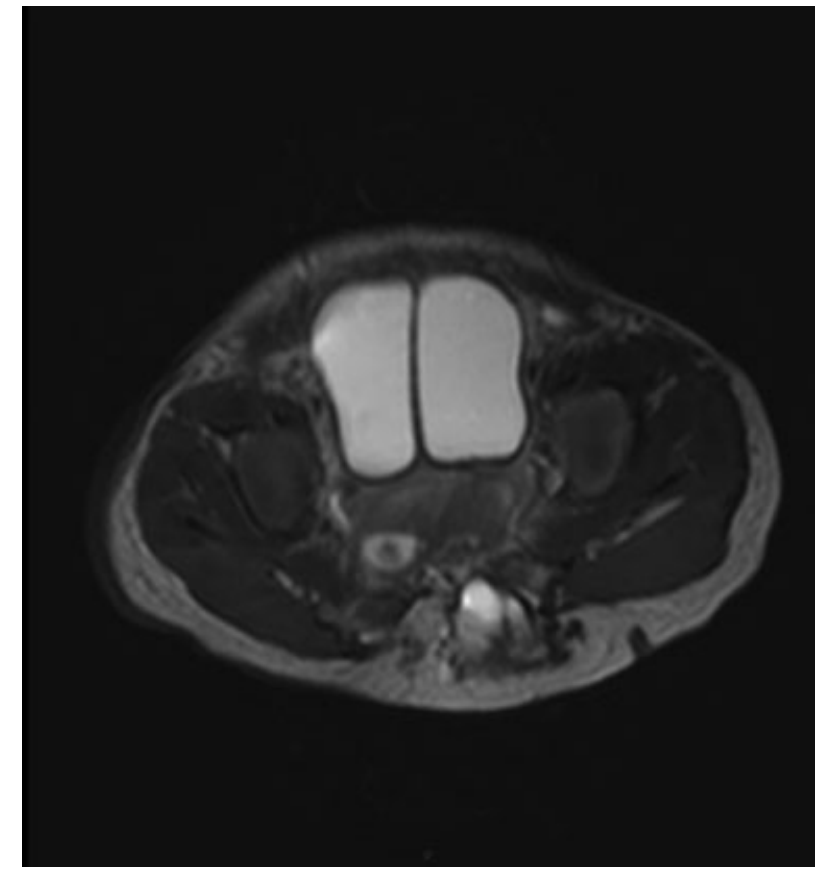

Fig. $4 \mathrm{MRI}$ of abdomen showing the two hemibladders with a midline sagittal septum.

normal bladder neck and no trigone. The left ureteral orifice was wide and a remnant of verumontanum was identified above the external sphincter. The proximal penoscrotal urethra led to a small right hemibladder with a normal bladder neck and no trigone. No verumontanum was found and the right ureteral orifice was normal. Cystometric study revealed a stable bladder with normal compliance. Each hemibladder capacity was $25 \mathrm{~mL}$ and the overall volume was normal for the baby's age. The contractions of hemibladders were asyn-

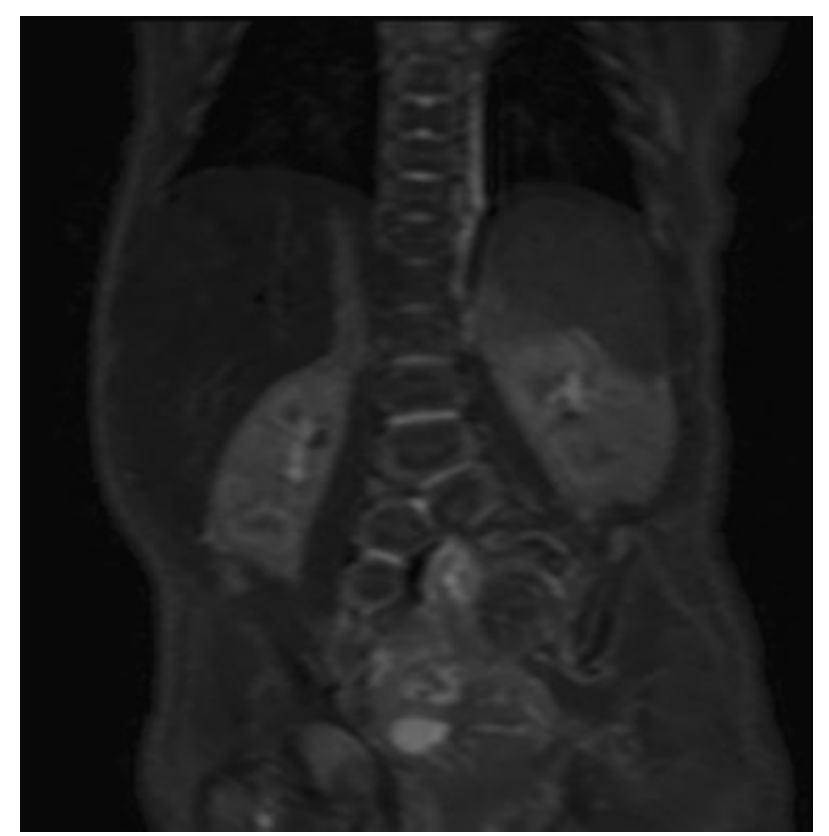

Fig. 5 Coronal MRI of the abdomen showing split of the spinal vertebrae below the dorsolumbar junction. chronous. Magnetic resonance imaging of abdomen and spine, performed under sedation, showed duplication of the bladder with a midline sagittal septum ( - Fig. 4), duplicated urethras, and splitting of the spinal vertebrae below the dorsolumbar junction ( $>$ Fig. 5) with tethering of cord. Due to a split in the vertebra, the thecal sac was exposed both anteriorly (to the abdomen) and posteriorly with a large lipomyelomeningocele.

The baby was formula fed since birth and had daily soft bowel movements. He gained weight and his developmental milestones were normal. His extremity sensory and motor functions were intact.

Following initial work-up and assessment, the case was discussed in a multidisciplinary working group involving a pediatric surgeon, pediatric urologist, and a pediatric neurosurgeon, to maximize management effectiveness. It was agreed that no immediate surgical intervention was required. At age 6 months, he started straining while stooling. On examination, both perineal fistulae accommodated a Hegar \#7 only. At that point, it was clear that the baby's only functional concern was the exacerbating constipation as a result of the relative distal obstruction. The perineal fistulae were tight and it was our impression that daily dilations would not relieve the constipation. The baby was taken for laparotomy on a semielective basis, to better study the colon anatomy and construct colostomies. Exploration revealed a tubular duplication of the colon with all layers of colon wall up to the level of the hepatic flexure with no other intraabdominal pathology (-Fig. 6). Duplicated colons shared a common blood supply. A duplicated appendix was not observed. The colon was resected from the level of the sigmoid colon to the transverse colon. This was performed to reduce the length of the duplicated colon which might cause future motility issues. We also found one lumen better than the other, and did not want any future obstruction caused by the second lumen, particularly considering that antegrade flushes would likely be needed in the future, and we did not want one lumen cleaned well and the other cleaned less well which would lead to soiling. Colostomy was fashioned resulting in two stoma openings, a functioning colostomy and a mucous fistula. Each stoma contained two lumens divided by a common wall. The common wall of the distal duplicated segment was stapled to create a single lumen below the peritoneal reflection. At age 11 months, the lipomyelomeningocele was removed and the cord was untethered. At age 1.5 years, a posterior anorectoplasty of the duplicated perineal fistulae was performed. Nice perineal muscles were observed. During the procedure, both fistulae were mobilized to the level of the previously constructed rectal reservoir (-Fig. 7). Then the distal separated rectal lumens were opened and joined to make a single rectum by closing the anterior and posterior walls along their entire length. The neoanus was placed in the anal dimple after splitting it. The muscles were located lateral to the fistulae on both sides and were brought to the midline on both sides of the single rectum and neoanus. The anorectoplasty was then completed. The postoperative period was uneventful and the baby was discharged for further ambulatory follow-up. 


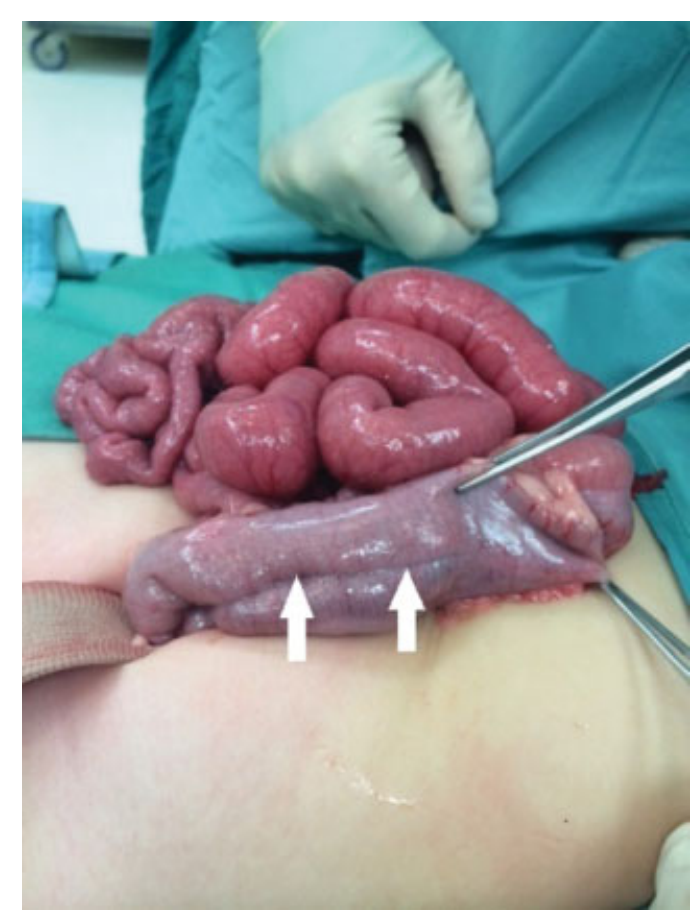

Fig. 6 On laparotomy, the duplicated rectum and colon shared a common wall (arrows).

\section{Discussion}

CDS is a rare entity that describes the association between anomalies involving caudal structures. Following an extensive work-up of all systems involved, the main consideration is to decide upon surgical strategy. In such complex cases, a multidisciplinary team approach involving surgery, urology,

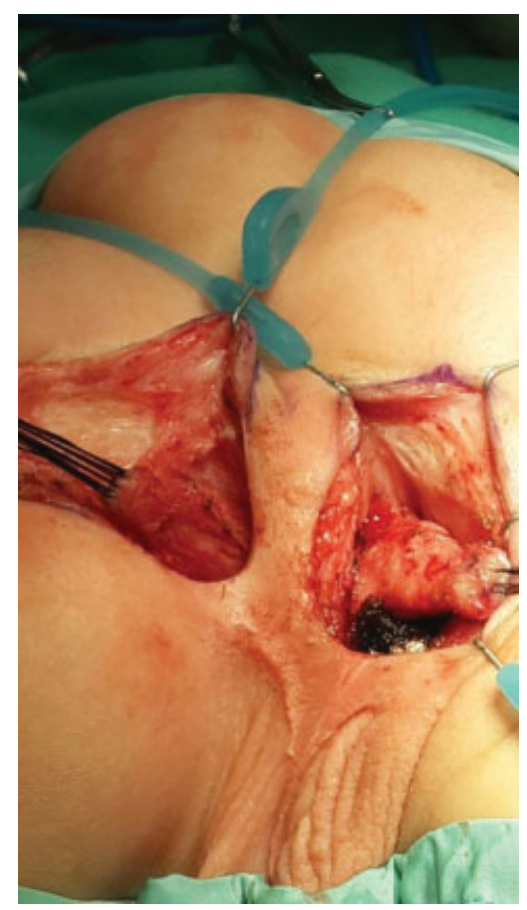

Fig. 7 Mobilization of perineal fistulae. and neurosurgery is warranted. This implies a staged correction of the varied malformations. The management plan must address important factors such as timing and sequence of the repair, patient's benefit from the intervention, and risk of contaminating the sterile surgical field by combining operations of different disciplines.

In our case, the most pressing issue was the tight perineal orifices. Although the baby was stooling daily, it was clear that he was developing constipation since stooling was accompanied by straining. In such cases, the surgeon should order a plain abdominal X-ray that will show whether the child is accumulating stool. Recurrent fecal impaction might result in extreme rectal dilatation that will possibly require rectal tapering during the rectal repair. We consider this a preventable complication. The surgeon should be alert to this development and avoid constipation with stool softeners and plan for colostomies at an early stage.

Colonic duplication is an uncommon malformation that usually presents in early childhood. Treatment approaches range from conservative expectant management for asymptomatic cases $^{4}$ to preventive strategy of resection of duplicated colon to avoid complications such as colon stricture and obstruction. ${ }^{5}$ Resection of the duplicated colon is feasible when the blood supply is duplicated as well. When the duplicated colons share a common wall, a septotomy is a practical option. ${ }^{6}$ In cases of duplication of both colon and rectum, colostomies as well as mucous fistulae should be constructed rather than a Hartmann pouch. These will also facilitated further radiological investigation of both colon limbs. A distal colostogram performed through the mucous fistulae will deliver valuable anatomic information prior to the definitive repair that would serve in preventing urologic injuries. ${ }^{7,8}$

Rectal duplications accompanying anorectal malformations are rare and in our case should be approached both abdominally and posterior sagittally. Since the duplications shared a common wall, a proximal septotomy was performed, converting the two rectums into a single reservoir. Through a posterior incision, as a second stage, we were able to mobilize both rectums proximally to the level of the rectal reservoir. The rectums were then joined to a single lumen and the anorectoplasty was completed.

Duplication of the spine is a rare anomaly ${ }^{9}$ and even more exceptional is the association with lipomyelomeningocele. ${ }^{10}$ While most patients with duplication of the spine have an associated neurologic deficit, some can be neurologically intact. $^{11,12}$ Our patient had normal sensory and motor function of his lower limbs and normal bladder function on cystometric studies.

Operative intervention of closing the myelomeningocele and detethering of the cord should be directed toward preserving neurological function. During the procedure, extreme caution should be employed not to injure the rectum. Such injury might complicate the procedure by cerebrospinal fluid infection. Diverting the colon prior to neurosurgical intervention decreases the hazards of such a complication. Performing the neurosurgical repair prior to the perineal repair clears the lipomeningomyelocele from the lower buttock area and thus enables us to perform a posterior sagittal incision. 
Despite the clearly malformed lower urinary tract anatomy, the bladder function remained normal. The mere finding of a duplicated bladder is not an indication for surgical intervention. ${ }^{13}$ Operation, if indicated, should aim at renal preservation and achieving urine continence. When a neurologic crossover is demonstrated, where one bladder contraction is transmitted to the other, a suggested management comprises excision of the common bladder wall and approximation of domes. ${ }^{14}$ This maneuver would synchronize hemibladder contractions. In our case, this bladder reconstruction as well as a hypospadias repair is planned with joining both urethras to create a common path.

For patients who hold poor prognosis for bowel control, a Malone procedure ${ }^{15}$ should be considered during the concluding surgical procedure of closure of the colostomies. Such a procedure must be coordinated with urology in case a Mitrofanoff procedure is considered. In such case, the appendix can be spilt for both purposes using the "split appendix technique." ${ }^{16}$ In cases of appendiceal duplication, care should be exploited to preserve both appendices that may turn out to be a valuable resource for the constructive operation.

In the presented case, the first surgical step was a sigmoid resection of the duplication, creation of stomas and septotomy of the common wall from the mucous fistulae down to the pelvis. The second step was a neurosurgery repair followed by a posterior anorectoplasty to create one rectum. Next step will be comprised of managing the proximal duplicated colon and restoring bowel continuity. This procedure will be combined with urology to merge the domes of the two hemibladder, thus achieving synchronized contractions. The final procedure will be a urethral repair.

\section{Summary}

These rare cases of CDS mandate carful investigation and work-up, bearing in mind the complexity and multifaceted nature of the syndrome. The surgical correction should be planned as a multidisciplinary staged approach involving urethral, bladder, colorectal and neurosurgical reconstructions, all impacting the other and requiring a clear preestablished surgical strategy.

\section{Conflict of Interest}

None

\section{References}

1 Dominguez R, Rott J, Castillo M, Pittaluga RR, Corriere JN Jr. Caudal duplication syndrome. Am J Dis Child 1993;147(10):1048-1052

2 Acer T, Ötgün İ, Sağnak Akıllı M, Gürbüz EE, Güney LH, Hiçsönmez A. A newborn with caudal duplication and duplex imperforate anus. J Pediatr Surg 2013;48(5):E37-E43

3 Bajpai M, Das K, Gupta AK. Caudal duplication syndrome: more evidence for theory of caudal twinning. J Pediatr Surg 2004;39(2): 223-225

4 Jellali MA, Mekki M, Saad J, et al. Perinatally discovered complete tubular colonic duplication associated with anal atresia. J Pediatr Surg 2012;47(6):e19-e23

5 Kekez T, Augustin G, Hrstic I, et al. Colonic duplication in an adult who presented with chronic constipation attributed to hypothyroidism. World J Gastroenterol 2008;14(4):644-646

6 Fuchs JR, Clark K, Breckler FD, Rescorla FJ. Complete colonic duplication-a case report. J Pediatr Surg 2008;43(6):E11-E13

7 Gross GW, Wolfson PJ, Pena A. Augmented-pressure colostogram in imperforate anus with fistula. Pediatr Radiol 1991;21(8): 560-562

8 Hong AR, Acuña MF, Peña A, Chaves L, Rodriguez G. Urologic injuries associated with repair of anorectal malformations in male patients. J Pediatr Surg 2002;37(3):339-344

9 Capasso G, Maffulli N. Dorsolumbar spine duplication. Acta Orthop Belg 1992;58(3):343-345

10 Yiğit H, Özdemir HM, Yurduseven E. Duplication of spine with hemi-lipomyelomeningocele. Eur Spine J 2013;22(Suppl 3): S487-S490

11 Incesu L, Karaismailoglu TN, Selcuk MB. Neurologically normal complete asymmetric lumbar spine duplication. AJNR Am J Neuroradiol 2004;25(5):895-896

12 Cebesoy O, Mete A, Karsli B. Complete lumbar spine duplication in a neurologically intact man. J Spinal Cord Med 2009;32(1):99-102

13 Abrahamson J. Double bladder and related anomalies: clinical and embryological aspects and a case report. $\mathrm{Br} \mathrm{J}$ Urol 1961; 33:195-214

14 Wisenbaugh ES, Palmer BW, Kropp BP. Successful management of a completely duplicated lower urinary system. J Pediatr Urol 2010; 6(3):315-317

15 Malone PS, Ransley PG, Kiely EM. Preliminary report: the antegrade continence enema. Lancet 1990;336(8725):1217-1218

16 VanderBrink BA, Cain MP, Kaefer M, Meldrum KK, Misseri R, Rink RC. Split-appendix technique for simultaneous appendicovesicostomy and appendicocecostomy. J Pediatr Surg 2011;46(1): 259-262 